\title{
Computing and Listing of Number of Possible m-sequence Generators of Order n
}

\author{
A. Ahmad ${ }^{1 *}$, S. S. Al-Busaidi', A. Al Maashri', M. Awadalla', M. A. K. Rizvi ${ }^{2}$ and N. Mohanan ${ }^{2}$ \\ 'Faculty, Department of Electrical and Computer Engineering, College of Engineering, Sultan Qaboos \\ University, P. O. Box 33, Postal Code 123; Muscat, Sultanate of Oman; afaq@squ.edu.om; \\ albusaid@squ.edu.om, amaashari@squ.edu.om,medhatha@squ.edu.om \\ ${ }^{2}$ M.Sc. Engineering Student, Department of Electrical and Computer Engineering, College of \\ Engineering, SultanQaboos University, P. O. Box 33, Postal Code 123; Muscat, Sultanate of Oman; \\ m099399@squ.edu.om,m100202@squ.edu.om
}

\begin{abstract}
Design of maximal length sequence (m-sequence) generators of order $n$ has many controlling parameters. In the design process of the generators it is essential to ensure that the generator characteristic polynomial corresponds to a primitive polynomial. The complexity of the search problem of primitive polynomials of order $\mathrm{n}$ grows as $\mathrm{n}$ increases and hence restricts the listing of all parameters of $\mathrm{m}$-sequence generators of order $\mathrm{n}$. This paper presents a computational procedure to determine the number of possible generators of order $\mathrm{n}$. The paper provides a list of all possible $\mathrm{m}$-sequence generators for up to $\mathrm{n}=100$.
\end{abstract}

Keywords: m-Sequence, LFSR, Primitive Polynomial, Prime Factors, Mersenne Numbers, MATLAB.

\section{Introduction and Problem Definition}

Maximal length sequences ( $\mathrm{m}$-sequence) are also known as Pseudorandom Noise (PN) sequences. Maximal length sequences are of great importance in a variety of applications such as Direct Sequence Spread Spectrum (DSSS), Built-in Self-Test (BIST), Decryption - Encryption System (DES) and error detection, just to mention a few [1-12].

Systems in these applications typically use the basic hardware named Linear Feedback Shift Register (LFSR) to generate $\mathrm{m}$-sequences [1-13]. A simple explanation of the LFSR structure and operation is given with respect to the structure shown in Figure 1 as follows. As shown in Figure 1 an LFSR is made up of two parts. These parts are a shift register and a feedback function. The shift registers, which can store one bit, are D - type Flip-Flops (FFs) that are connected as a chain. Moreover, each D-FF is also connected to a clock. At each clock cycle, a new bit is loaded into the first shift register, $\mathrm{D}-\mathrm{FF}_{1}$, of the D-FF chain. Considering that $\mathrm{D}-\mathrm{FF}_{1}$ is the leftmost register then the remaining bits within all other shift registers are shifted to the neighboring right register at every clock cycle. Furthermore, there exists a feedback function, which is simply the Exclusive-OR (XOR) logic operation of a number of bits that are held within a prescribed number of registers. Registers that are involved in the feedback operation are all connected to the XOR operator. Alternatively, the sequence of connections which are involved in the XOR operation logic is referred to a sequence of feedback taps $\left(C_{0} C_{1} C_{2} \ldots \ldots C_{\mathrm{i}} \ldots C_{\mathrm{n}-1} C_{\mathrm{n}}\right)$. The updated left most bit state of D-FF ${ }_{1}$ is computed as a function of the existing feedback taps of the LFSR. The output of the LFSR is then read out at the output of rightmost shift register, one bit every clock cycle. Finally, the period of a shift register, $\mathrm{p}$, is the length of the output sequence before it starts repeating [1-17].

*Corresponding author:

A. Ahmad (afaq@squ.edu.om) 
Definition 1: An LFSR is a special type of Serial-In SerialOut (SISO) shift register that, when clocked, propagates bits from the least significant to the most significant bit position through its constituent neighbouring registers, one bit every clock cycle.

Figure 1 shows an $n$-bit SISO shift register. The key element of SISO shift registers is the D-type FFs. The sequence $\left\{q_{1}, q_{2}, \ldots q_{i}, \ldots q_{n-1}, q_{n}\right\}$ represents the states of the FFs $\left\{\mathrm{D}_{1}\right.$, $\left.\mathrm{D}_{2}, \ldots, \mathrm{D}_{\mathrm{i}}, \ldots \mathrm{D}_{n-1,}, \mathrm{D}_{n}\right\}$ respectively. Table 1 demonstrates an example of how LFSRs operates to generate an $\mathrm{m}$-sequence. The $\mathrm{m}$-sequence generator considered for this demonstration is shown in Figure 2.

Figure 2 depicts a 3-bit LFSR, which is constructed using an external XOR functional block. Note how the feedback - which is fed into the input to the first FF - is the result of exclusive-OR operation of the outputs of the second and third FFs. Table 1 visualizes the operation of the LFSR depicted in Figure 2. The table elaborates the next states (FF1_OUT, FF2_OUT, and FF3_OUT) and the output m-sequence $S_{i}$. The initial state of the LFSR is considered to be as follows, $q_{1}(0)=1, q_{2}(0)=0$, and $q_{3}$ $(0)=1$.

It is this feedback function that causes the LFSR to loop through a repetitive sequence of values. The choice of feedback connections, the initial state and the value of $n$, all determine the number of elements in a given sequence

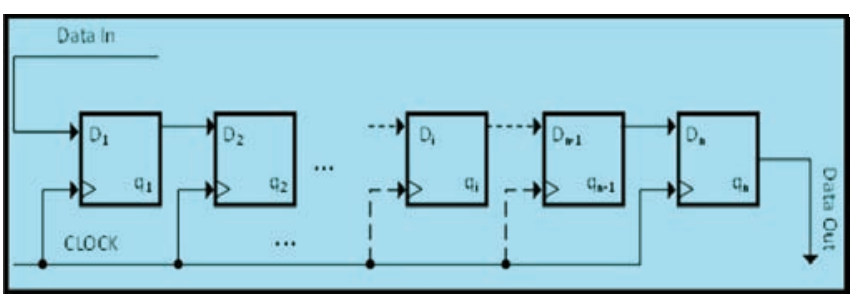

Figure 1. An n-bit SISO shift register.

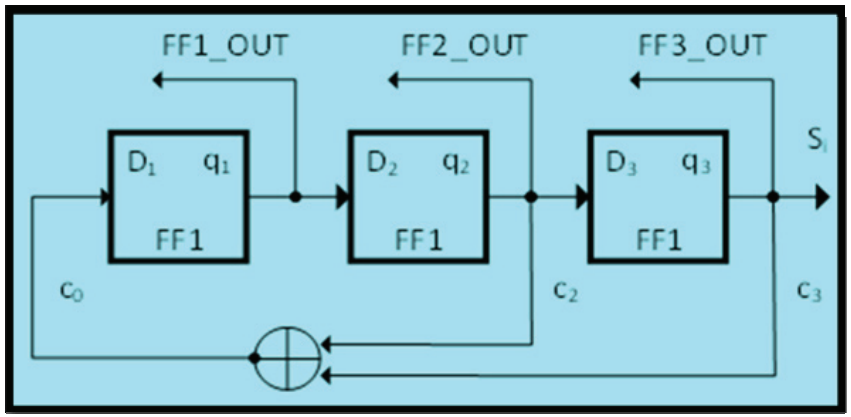

Figure 2. A 3-bit LFSR (External EOR type). before the sequence repeats. The number of elements, in turn, determines the sequence length. This length is known as the periodicity, $p$, of the LFSR $[2,4,6,18-24]$.

Definition 2: The period $p$ of an n-bit LFSR may vary from $p=1$ to $2^{n}-1$.

Definition 3: The sequence having length of $2^{n}-1$ is known as maximal length sequence ( $\mathrm{m}$-sequence).

Any binary sequence can be represented in a polynomial form over the Galois Field 2, GF(2). As such, the feedback connection vector of an LFSR can be represented by a polynomial that is technically referred to as a characteristic polynomial. Equations (1) define a general form of a characteristic polynomial which can be denoted as $\mathrm{D}(\mathrm{x})$.

$\mathrm{D}(x)=\left(c_{0} \times x^{0}\right)+\left(c_{1} \times x^{1}\right)+\ldots+\left(c_{\mathrm{n}} \times x^{\mathrm{n}}\right)$

For any LFSR structure of length $n$, a list of all characteristic polynomials can be generated to encompass every possible connection. To demonstrate this, Table 2 lists all the characteristic polynomials, NP, for an LFSR of length 3.

TheOrem 1: In an n-bit LFSR a sequence generator can be referred to as an m-sequence generator only if its characteristic polynomial is primitive.

Table 1. Next state $\mathrm{m}$-sequences for the structure of LFSR of Figure 2

\begin{tabular}{ccccc}
\hline Clock & $q_{1}$ & $q_{2}$ & $q_{3}$ & $S \mathbf{i}$ \\
& (FF1_OUT) & (FF2_OUT) & (FF__OUT) & \\
\hline 0 & 1 & 0 & 1 & \\
1 & 1 & 1 & 0 & \\
2 & 1 & 1 & 1 & \\
3 & 0 & 1 & 1 & $\ldots 11101001110$ \\
4 & 0 & 0 & 1 & \\
5 & 1 & 0 & 0 & \\
6 & 0 & 1 & 0 & Repeats \\
7 & 1 & 0 & 1 & \\
\hline
\end{tabular}

Table 2. Number of possible generators (NP) and number of possible $\mathrm{m}$-sequence generators in LFSR of Figure 2

\begin{tabular}{ccc}
\hline$n$ & $N P$ & $N P P$ \\
\hline 3 & 4 & 2 \\
& $1+\left(x^{3}\right)$ & $1+\left(x^{2}\right)+\left(x^{3}\right)$ \\
& $1+\left(x^{2}\right)+\left(x^{3}\right)$ & $1+(x)+\left(x^{2}\right)+\left(x^{3}\right)$ \\
& $1+(x)+\left(x^{3}\right)$ & \\
& $1+(x)+\left(x^{2}\right)+\left(x^{3}\right)$ & \\
\hline
\end{tabular}


Table 2 also demonstrates that out of the 4 possible characteristic polynomials only 2 can be used to generate $\mathrm{m}$-sequences. It can therefore be assumed that the number of primitive characteristic polynomials, NPP, is a subset of NP, by which the relation NPP $<$ NP should hold.

An interesting relation on the complexity search for primitive characteristic polynomials can also be established. As $n$ increases, the search complexity for primitive characteristic polynomials of order $n$ grows exponentially. Consequently, the search success decays similar to the exponential decay of a signal. The nature of this search success decay rate is depicted in Figure 3. The search of primitive polynomials from the list of all possible combinations of generator polynomials can be prohibitive for large n. To this extent, this paper presents a computational efficient procedure of determining $N P P$ when $\mathrm{n}$ is large.

\section{Controlling Parameters for m-sequence Generators and Mathematics}

The parameters governing the sequence period of a generator are: 1) the order $n, 2)$ the initial state, and 3) the used characteristic polynomial. Table 1 shows an example where $\mathrm{n}=3$, initial state: $\left[\mathrm{q}_{1}=1, \mathrm{q}_{2}=0\right.$, and $\left.\mathrm{q}_{3}=1\right]$, and characteristic polynomials:

$$
\begin{gathered}
1+\left(x^{3}\right), \\
1+\left(x^{2}\right)+\left(x^{3}\right), \\
1+(x)+\left(x^{3}\right), \text { and } \\
1+(x)+\left(x^{2}\right)+\left(x^{3}\right) .
\end{gathered}
$$

The sequence periods $(p)$ generated by the characteristic polynomials shown above are 3, 7, 7 and 4 , respectively. Note that each of the sequence generators is governed by a different state equation. In general, the state equation of a sequence generator is defined by Equation (2).

$$
q(t+1)=[A] * q(t)
$$

where, system matrix of the generator is $[A]$, while $\mathrm{q}(t+1)$ and $\mathrm{q}(t)$ are next and present states of the generator, respectively. The structure of matrix $[A]$ for an $n$ order can be defined in Equation (3).

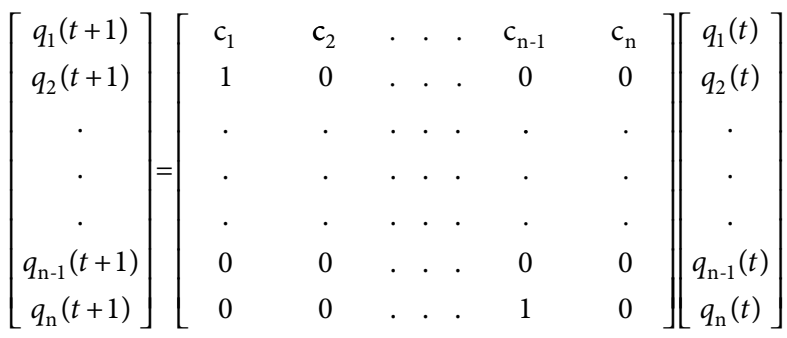

$$
\begin{aligned}
& \text { where } \quad c_{j}=\left\{\begin{array}{l}
0 \text { or } 1, \text { for } 1 \leq \mathrm{j} \leq n-1 \\
1, \quad \text { for } j=n
\end{array}\right.
\end{aligned}
$$

In Equation (4), the values of $c_{j}$ show the existence or absence of a feedback connection from the $j$-th stage of the LFSR. Equation (2) can be written as:

$$
[q(t+1)]=[A][q(t)]
$$

If $[q]=[q(0)]$ represents a particular initial loading of the LFSR, then the sequence of states through which the LFSR will pass during successive times is given by

$$
[q(t)],[A][q(t)],[A]^{2}[q(t)],[A]^{3}[q(t)], \ldots
$$

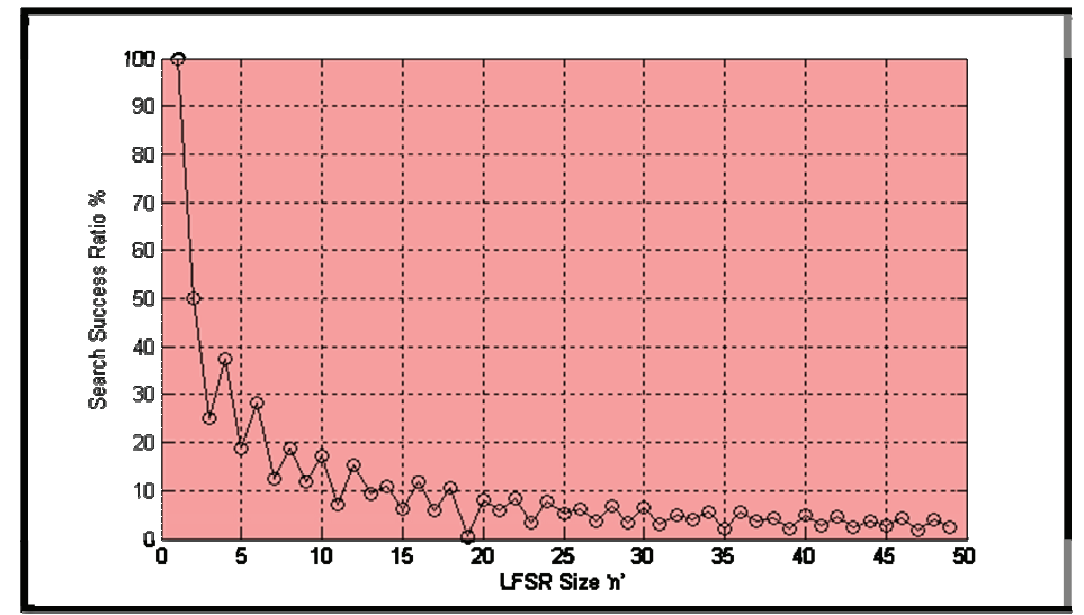

Figure 3. Search success rate for $\mathrm{m}$-sequence generator in an-bit LFSR. 
Let the matrix 'period' be the smallest integer $\mathrm{p}$ for which $[A]^{p}=I$, where $I$ is an identity matrix. Then $[A]^{p}$ $[q(t)]=[q(t)]$ for any non-zero initial vector $[q(0)]$, indicating the 'cycle length (or period)' of the LFSR is $p$.

The cycle length for $[q(0)]=0$ is always 1 , independent of matrix $[A]$. Thus, on the basis of this property of periodicity of LFSR and Equation (5), it follows that:

$$
[q(t)]=[q(t+p)]=[A]^{p}[q(t)]
$$

The following corollaries cover the periodicity properties of an LFSR and its relation with its corresponding primitive characteristic polynomial. These are used in the proposed algorithm.

Corollary 1: If $p=m=2^{n}-1$ is a prime number, then the characteristic polynomial corresponds to that connection of LFSR will be primitive, if and only if $[A]^{m}=1$.

Corollary 2: If $p=m=2^{n}-1$ is not a prime number, and $[A]^{p i}=1$, where $p_{i}$ is a divisor of $p$, then the characteristic polynomial corresponds to that connection of LFSR cannot be primitive.

The determination of the primitive polynomial comprises of two folds; 1) the use of the Euler phi-function $\varphi($.$) , and$ 2 ) the search for primes. The Euler function has the property that its value for an integer $m$ is the product of the values of the Euler phi-function at the prime powers that occur in the factorization of $m$. The Euler phi-function can be computed on the basis of the prime factorization of $m$. The following theorems and Lemma are embodied in the proposed algorithm for finding primitive polynomials.

LEMMA 1: There exists (exist) a prime divisor (or divisors) for every positive integer greater than one.

THEOREM 2: If $m$ is a composite integer, then $m$ has a prime factor not exceeding the prime integer value of $\sqrt{m}$.

Theorem 3: Let $m=p_{1}^{a_{1}} p_{2}^{a_{2}} \ldots p_{i}^{a_{i}} \ldots p_{k}^{a_{k}}$ be the prime $\left(p_{i}\right)$ of power $\left(a_{i}\right)$ factorization of the positive integer $m$. Then

$$
\varphi(\mathrm{m})=\mathrm{m}\left(1-1 / p_{1}\right)\left(1-1 / p_{2}\right) \ldots\left(1-1 / p_{k}\right)
$$

TheOrem 4: The total number of possible primitive polynomials (NPP) of order $n$ is given by

$$
N P P=\frac{(m)}{n}
$$

\section{Computing Factors of $m,((m)$ and NPP [25-32]}

Two algorithms - designated A.1 and A.2 - are used to compute factors of $m, \varphi(m)$, and NPP. These two algorithms are presented in pseudo code format as follows:

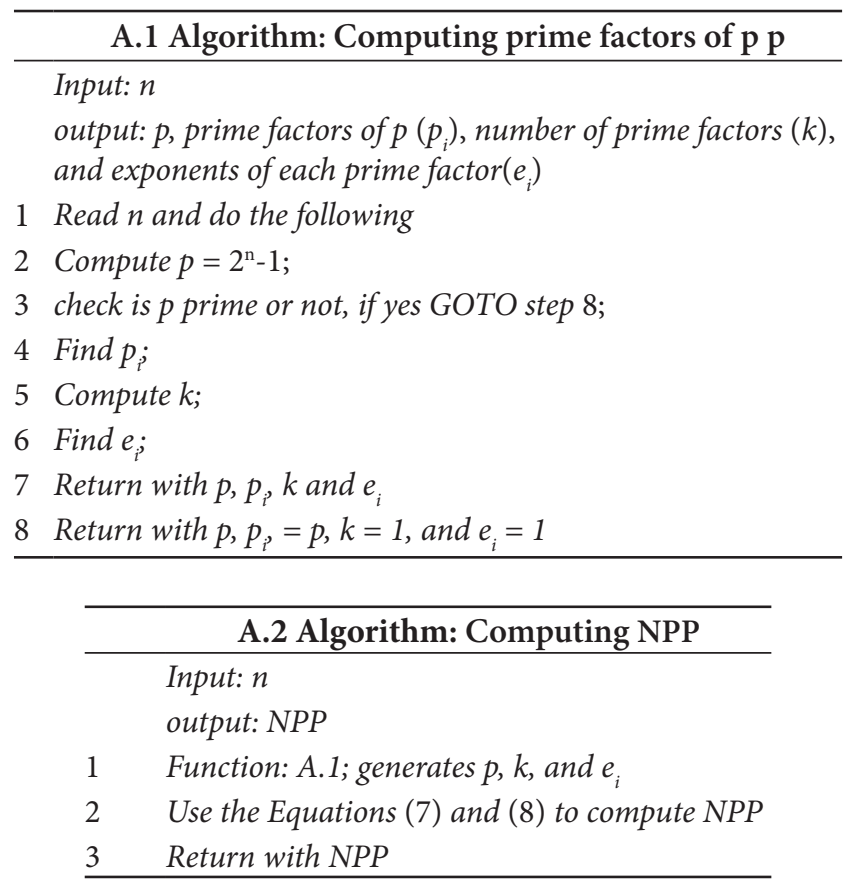

The factors for $n=1$ to 100 are listed in Table 3 and 4; whereas the values of $\varphi(m)$ and NPP are shown in Tables 5 and 6. Factorisations are given from smallest to largest factor, with a period '. in the table ' $\mathrm{f}$ ' indicates the number of factors.

\section{Conclusions}

We developed an algorithm and succeeded in getting the values of NPP for large values of $n$. This paper represent our efforts in transforming our observations into algorithms that are capable of determining the values of NPP for large $n$. Using these algorithm, we generated NPP lookup tables, which show the factors of $m$-sequence period $m$ and $N P P$. As an example let $n=4$, gives $N P$ as 4 while NPP is computed as 2 . The factors for $m=15$ suggests that the periods of sequence generators of size $n=$ 4 may be any value from factors $\{1,3,5,15\}$. Only two of the generators are giving $\mathrm{m}$-sequence. These tables offer fruitful information that can be utilized to judge whether 
Table 3. The Factors of $m$ ( for $n=1$ to 50)[Exponents are represented with ' $\wedge$ ']

\begin{tabular}{|c|c|c|c|c|}
\hline$n$ & $m$ & $p$ & $f$ & Factors \\
\hline 1 & 1 & $\mathrm{Y}$ & 1 & 1 \\
\hline 2 & 3 & $\mathrm{Y}$ & 1 & 3 \\
\hline 3 & 7 & $\mathrm{Y}$ & 1 & 7 \\
\hline 4 & 15 & $\mathrm{~N}$ & 2 & $3 \times 5$ \\
\hline 5 & 31 & $\mathrm{Y}$ & 1 & 31 \\
\hline 6 & 63 & $\mathrm{~N}$ & 3 & $3 \times 3 \times 7 \rightarrow 3^{\wedge} 2.7$ \\
\hline 7 & 127 & $\mathrm{Y}$ & 1 & 127 \\
\hline 8 & 255 & $\mathrm{~N}$ & 3 & $3 \times 5 \times 17$ \\
\hline 9 & 511 & $\mathrm{~N}$ & 2 & $7 \times 73$ \\
\hline 10 & 1023 & $\mathrm{~N}$ & 3 & $3 \times 11 \times 31$ \\
\hline 11 & 2047 & $\mathrm{~N}$ & 2 & $23 \times 89$ \\
\hline 12 & 4095 & $\mathrm{~N}$ & 5 & $3 \times 3 \times 5 \times 7 \times 13 \rightarrow 3^{\wedge} 2.5 .7 .13$ \\
\hline 13 & 8191 & $\mathrm{Y}$ & 1 & 8191 \\
\hline 14 & 16384 & $\mathrm{~N}$ & 3 & $3 \times 43 \times 127$ \\
\hline 15 & 32768 & $\mathrm{~N}$ & 3 & $7 \times 31 \times 151$ \\
\hline 16 & 65536 & $\mathrm{~N}$ & 4 & $3 \times 5 \times 17 \times 257$ \\
\hline 17 & 131072 & $\mathrm{Y}$ & 1 & 131071 \\
\hline 18 & 262144 & $\mathrm{~N}$ & 6 & $3 \times 3 \times 3 \times 7 \times 19 \times 73 \rightarrow 3^{\wedge} 3.7 .19 .73$ \\
\hline 19 & 524288 & $\mathrm{Y}$ & 1 & 524287 \\
\hline 20 & 1048576 & $\mathrm{~N}$ & 6 & $3 \times 5 \times 5 \times 11 \times 31 \times 41 \rightarrow 3.5^{\wedge} 2.11 .31 .41$ \\
\hline 21 & 2097152 & $\mathrm{~N}$ & 4 & $7 \times 7 \times 127 \times 337 \rightarrow 7^{\wedge} 2.127 .337$ \\
\hline 22 & 4194304 & $\mathrm{~N}$ & 4 & $3 \times 23 \times 89 \times 683$ \\
\hline 23 & 8388608 & $\mathrm{Y}$ & 2 & $47 \times 178481$ \\
\hline 24 & 16777216 & $\mathrm{~N}$ & 7 & $3 \times 3 \times 5 \times 7 \times 13 \times 17 \times 241 \rightarrow 3^{\wedge} 2.5 .7 .13 .17 .241$ \\
\hline 25 & 33554432 & $\mathrm{~N}$ & 3 & $31 \times 601 \times 1801$ \\
\hline 26 & 67108864 & $\mathrm{~N}$ & 3 & $3 \times 2731 \times 8191$ \\
\hline 27 & 134217728 & $\mathrm{~N}$ & 3 & $7 \times 73 \times 262657$ \\
\hline 28 & 268435456 & $\mathrm{~N}$ & 6 & $3 \times 5 \times 29 \times 43 \times 113 \times 127$ \\
\hline 29 & 536870912 & $\mathrm{Y}$ & 3 & $233 \times 1103 \times 2089$ \\
\hline 30 & 1073741824 & $\mathrm{~N}$ & 7 & $3 \times 3 \times 7 \times 11 \times 31 \times 151 \times 331 \rightarrow 3 \wedge 2.7 .11 .31 .151 .331$ \\
\hline 31 & 2147483648 & $\mathrm{Y}$ & 1 & 2147483647 \\
\hline 32 & 4294967296 & $\mathrm{~N}$ & 5 & $3 \times 5 \times 17 \times 257 \times 65537$ \\
\hline 33 & 8589934592 & $\mathrm{~N}$ & 4 & $7 \times 23 \times 89 \times 599479$ \\
\hline 34 & 17179869184 & $\mathrm{~N}$ & 3 & $3 \times 43691 \times 131071$ \\
\hline 35 & 34359738368 & $\mathrm{~N}$ & 4 & $31 \times 71 \times 127 \times 122921$ \\
\hline 36 & 68719476736 & $\mathrm{~N}$ & 10 & $3 \times 3 \times 3 \times 5 \times 7 \times 13 \times 19 \times 37 \times 73 \times 109 \rightarrow 3 \wedge 3.5 .7 .13 .19 .37 .73 .109$ \\
\hline 37 & $1.37439 \mathrm{E}+11$ & $\mathrm{Y}$ & 2 & $223 \times 616318177$ \\
\hline 38 & $2.74878 \mathrm{E}+11$ & $\mathrm{~N}$ & 3 & $3 \times 174763 \times 524287$ \\
\hline 39 & $5.49756 \mathrm{E}+11$ & $\mathrm{~N}$ & 4 & $7 \times 79 \times 8191 \times 121369$ \\
\hline 40 & $1.09951 \mathrm{E}+12$ & $\mathrm{~N}$ & 8 & $3 \times 5 \times 5 \times 11 \times 17 \times 31 \times 41 \times 61681 \rightarrow 3.5^{\wedge} 2.11 .17 .31 .41 .61681$ \\
\hline 41 & $2.19902 \mathrm{E}+12$ & $\mathrm{Y}$ & 2 & $13367 \times 164511353$ \\
\hline 42 & $4.39805 \mathrm{E}+12$ & $\mathrm{~N}$ & 8 & $3 \times 3 \times 7 \times 7 \times 43 \times 127 \times 337 \times 5419 \rightarrow 3^{\wedge} 2.7^{\wedge} 2.43 .127 .337 .5419$ \\
\hline 43 & $8.79609 \mathrm{E}+12$ & $\mathrm{Y}$ & 3 & $431 \times 9719 \times 2099863$ \\
\hline 44 & $1.75922 \mathrm{E}+13$ & $\mathrm{~N}$ & 7 & $3 \times 5 \times 23 \times 89 \times 397 \times 683 \times 2113$ \\
\hline 45 & $3.51844 \mathrm{E}+13$ & $\mathrm{~N}$ & 6 & $7 \times 31 \times 73 \times 151 \times 631 \times 23311$ \\
\hline 46 & $7.03687 \mathrm{E}+13$ & $\mathrm{~N}$ & 4 & $3 \times 47 \times 178481 \times 2796203$ \\
\hline
\end{tabular}


Table 3. (Continued)

\begin{tabular}{lllcl}
47 & $1.40737 \mathrm{E}+14$ & $\mathrm{Y}$ & 3 & $2351 \times 4513 \times 13264529$ \\
48 & $2.81475 \mathrm{E}+14$ & $\mathrm{~N}$ & 10 & $3 \times 3 \times 5 \times 7 \times 13 \times 17 \times 97 \times 241 \times 257 \times 673$ \\
& & & & $\rightarrow 3 \wedge 2.5 .7 .13 .17 .97 .241 .257 .673$ \\
49 & $5.6295 \mathrm{E}+14$ & $\mathrm{~N}$ & 2 & $127 \times 4432676798593$ \\
50 & $1.1259 \mathrm{E}+15$ & $\mathrm{~N}$ & 7 & $3 \times 11 \times 31 \times 251 \times 601 \times 1801 \times 4051$ \\
\hline
\end{tabular}

Table 4. The Factors of $m$ (for $n=51$ to 100) [Exponents are represented with`^’]

\begin{tabular}{|c|c|c|c|c|}
\hline $\bar{n}$ & $m$ & $p$ & $f$ & Factors \\
\hline 51 & $2.2518 \mathrm{E}+15$ & $\mathrm{~N}$ & 5 & $7 \times 103 \times 2143 \times 11119 \times 131071$ \\
\hline 52 & $4.5036 \mathrm{E}+15$ & $\mathrm{~N}$ & 7 & $3 \times 5 \times 53 \times 157 \times 1613 \times 2731 \times 8191$ \\
\hline 53 & $9.0072 \mathrm{E}+15$ & $\mathrm{Y}$ & 3 & $6361 \times 69431 \times 20394401$ \\
\hline 54 & $1.80144 \mathrm{E}+16$ & $\mathrm{~N}$ & 9 & $\begin{array}{l}3 \times 3 \times 3 \times 3 \times 7 \times 19 \times 73 \times 87211 \times 262657 \\
\rightarrow 3^{\wedge} 4.7 .19 .73 .87211 .262657\end{array}$ \\
\hline 55 & $3.60288 \mathrm{E}+16$ & $\mathrm{~N}$ & 6 & $23 \times 31 \times 89 \times 881 \times 3191 \times 201961$ \\
\hline 56 & $7.20576 \mathrm{E}+16$ & $\mathrm{~N}$ & 8 & $3 \times 5 \times 17 \times 29 \times 43 \times 113 \times 127 \times 15790321$ \\
\hline 57 & $1.44115 \mathrm{E}+17$ & $\mathrm{~N}$ & 4 & $7 \times 32377 \times 524287 \times 1212847$ \\
\hline 58 & $2.8823 \mathrm{E}+17$ & $\mathrm{~N}$ & 6 & $3 \times 59 \times 233 \times 1103 \times 2089 \times 3033169$ \\
\hline 59 & $5.76461 \mathrm{E}+17$ & $\mathrm{Y}$ & 2 & $179951 \times 3203431780337$ \\
\hline 60 & $1.15292 \mathrm{E}+18$ & $\mathrm{~N}$ & 13 & $\begin{array}{l}3 \times 3 \times 5 \times 5 \times 7 \times 11 \times 13 \times 31 \times 41 \times 61 \times 151 \times 331 \times 1321 \\
\rightarrow 3^{\wedge} 2.5^{\wedge} 2.7 .11 .13 .31 .41 .61 .151 .331 .1321\end{array}$ \\
\hline 61 & $2.30584 \mathrm{E}+18$ & $\mathrm{Y}$ & 1 & 2305843009213693951 \\
\hline 62 & $4.61169 \mathrm{E}+18$ & $\mathrm{~N}$ & 3 & $3 \times 715827883 \times 2147483647$ \\
\hline 63 & $9.22337 \mathrm{E}+18$ & $\mathrm{~N}$ & 7 & $\begin{array}{l}7 \times 7 \times 73 \times 127 \times 337 \times 92737 \times 649657 \\
7 \wedge 2.73 .127 .337 .92737 .649657\end{array}$ \\
\hline 64 & $1.84467 \mathrm{E}+19$ & $\mathrm{~N}$ & 7 & $3 \times 5 \times 17 \times 257 \times 641 \times 65537 \times 6700417$ \\
\hline 65 & $3.68935 \mathrm{E}+19$ & $\mathrm{~N}$ & 3 & $31 \times 8191 \times 145295143558111$ \\
\hline 66 & $7.3787 \mathrm{E}+19$ & $\mathrm{~N}$ & 9 & $\begin{array}{l}3 \times 3 \times 7 \times 23 \times 67 \times 89 \times 683 \times 20857 \times 599479 \\
\rightarrow 3^{\wedge} 2.7 .23 .67 .89 .683 .20857 .599479\end{array}$ \\
\hline 67 & $1.47574 \mathrm{E}+20$ & $\mathrm{Y}$ & 2 & $193707721 \times 761838257287$ \\
\hline 68 & $2.95148 \mathrm{E}+20$ & $\mathrm{~N}$ & 7 & $3 \times 5 \times 137 \times 953 \times 26317 \times 43691 \times 131071$ \\
\hline 69 & $5.90296 \mathrm{E}+20$ & $\mathrm{~N}$ & 4 & $7 \times 47 \times 178481 \times 10052678938039$ \\
\hline 70 & $1.18059 \mathrm{E}+21$ & $\mathrm{~N}$ & 9 & $3 \times 11 \times 31 \times 43 \times 71 \times 127 \times 281 \times 86171 \times 122921$ \\
\hline 71 & $2.36118 \mathrm{E}+21$ & $\mathrm{Y}$ & 3 & $228479 \times 48544121 \times 212885833$ \\
\hline 72 & $4.72237 \mathrm{E}+21$ & $\mathrm{~N}$ & 14 & $\begin{array}{l}3 \times 3 \times 3 \times 5 \times 7 \times 13 \times 17 \times 19 \times 37 \times 73 \times 109 \times 241 \times 433 \times 38737 \\
\rightarrow 3^{\wedge} 3.5 .7 .13 .17 .19 .37 .73 .109 .241 .433 .38737\end{array}$ \\
\hline 73 & $9.44473 \mathrm{E}+21$ & $\mathrm{Y}$ & 3 & $439 \times 2298041 \times 9361973132609$ \\
\hline 74 & $1.88895 \mathrm{E}+22$ & $\mathrm{~N}$ & 5 & $3 \times 223 \times 1777 \times 25781083 \times 616318177$ \\
\hline 75 & $3.77789 \mathrm{E}+22$ & $\mathrm{~N}$ & 7 & $7 \times 31 \times 151 \times 601 \times 1801 \times 100801 \times 10567201$ \\
\hline 76 & $7.55579 \mathrm{E}+22$ & $\mathrm{~N}$ & 7 & $3 \times 5 \times 229 \times 457 \times 174763 \times 524287 \times 525313$ \\
\hline 77 & $1.51116 \mathrm{E}+23$ & $\mathrm{~N}$ & 4 & $23 \times 89 \times 127 \times 581283643249112959$ \\
\hline 78 & $3.02231 \mathrm{E}+23$ & $\mathrm{~N}$ & 8 & $\begin{array}{l}3 \times 3 \times 7 \times 79 \times 2731 \times 8191 \times 121369 \times 22366891 \\
\rightarrow 3 \wedge 2.7 .79 .2731 .8191 .121369 .22366891\end{array}$ \\
\hline 79 & $6.04463 \mathrm{E}+23$ & $\mathrm{Y}$ & 3 & $2687 \times 202029703 \times 1113491139767$ \\
\hline 80 & $1.20893 \mathrm{E}+24$ & $\mathrm{~N}$ & 10 & $\begin{array}{l}3 \times 5 \times 5 \times 11 \times 17 \times 31 \times 41 \times 257 \times 61681 \times 4278255361 \\
\rightarrow 3.5^{\wedge} 2.11 .17 .31 .41 .257 .61681 .4278255361\end{array}$ \\
\hline 81 & $2.41785 \mathrm{E}+24$ & $\mathrm{~N}$ & 6 & $7 \times 73 \times 2593 \times 71119 \times 262657 \times 97685839$ \\
\hline 82 & $4.8357 \mathrm{E}+24$ & $\mathrm{~N}$ & 5 & $3 \times 83 \times 13367 \times 164511353 \times 8831418697$ \\
\hline 83 & $9.67141 \mathrm{E}+24$ & $\mathrm{Y}$ & 2 & $167 \times 57912614113275649087721$ \\
\hline
\end{tabular}


Table 4. (Continued)

\begin{tabular}{lllll}
84 & $1.93428 \mathrm{E}+25$ & $\mathrm{~N}$ & 14 & $\begin{array}{l}3 \times 3 \times 5 \times 7 \times 7 \times 13 \times 29 \times 43 \times 113 \times 127 \times 337 \times 1429 \times 5419 \times 14449 \\
\end{array}$ \\
85 & $3.86856 \mathrm{E}+25$ & $\mathrm{~N}$ & 3 & $31 \times 131071 \times 9520972806333758431$ \\
86 & $7.73713 \mathrm{E}+25$ & $\mathrm{~N}$ & 5 & $3 \times 431 \times 9719 \times 2099863 \times 2932031007403$ \\
87 & $1.54743 \mathrm{E}+26$ & $\mathrm{~N}$ & 6 & $7 \times 233 \times 1103 \times 2089 \times 4177 \times 9857737155463$ \\
88 & $3.09485 \mathrm{E}+26$ & $\mathrm{~N}$ & 10 & $3 \times 5 \times 17 \times 23 \times 89 \times 353 \times 397 \times 683 \times 2113 \times 2931542417$ \\
89 & $6.1897 \mathrm{E}+26$ & $\mathrm{Y}$ & 1 & 618970019642690137449562111 \\
90 & $1.23794 \mathrm{E}+27$ & $\mathrm{~N}$ & 13 & $3 \times 3 \times 3 \times 7 \times 11 \times 19 \times 31 \times 73 \times 151 \times 331 \times 631 \times 23311 \times 18837001$ \\
& & & & $\rightarrow 3 \wedge 3.7 .11 .19 .31 .73 .151 .331 .631 .23311 .18837001$ \\
91 & $2.47588 \mathrm{E}+27$ & $\mathrm{~N}$ & 5 & $127 \times 911 \times 8191 \times 112901153 \times 23140471537$ \\
92 & $4.95176 \mathrm{E}+27$ & $\mathrm{~N}$ & 9 & $3 \times 5 \times 47 \times 277 \times 1013 \times 1657 \times 30269 \times 178481 \times 2796203$ \\
93 & $9.90352 \mathrm{E}+27$ & $\mathrm{~N}$ & 3 & $7 \times 2147483647 \times 658812288653553079$ \\
94 & $1.9807 \mathrm{E}+28$ & $\mathrm{~N}$ & 6 & $3 \times 283 \times 2351 \times 4513 \times 13264529 \times 165768537521$ \\
95 & $3.96141 \mathrm{E}+28$ & $\mathrm{~N}$ & 5 & $31 \times 191 \times 524287 \times 420778751 \times 30327152671$ \\
96 & $7.92282 \mathrm{E}+28$ & $\mathrm{~N}$ & 13 & $3 \times 3 \times 5 \times 7 \times 13 \times 17 \times 97 \times 193 \times 241 \times 257 \times 673 \times 65537 \times 22253377$ \\
& & & & $\rightarrow 3 \wedge 2.5 .7 .13 .17 .97 .193 .241 .257 .673 .65537 .22253377$ \\
97 & $1.58456 \mathrm{E}+29$ & $\mathrm{Y}$ & 2 & $11447 \times 13842607235828485645766393$ \\
98 & $3.16913 \mathrm{E}+29$ & $\mathrm{~N}$ & 5 & $3 \times 43 \times 127 \times 4363953127297 \times 4432676798593$ \\
99 & $6.33825 \mathrm{E}+29$ & $\mathrm{~N}$ & 8 & $7 \times 23 \times 73 \times 89 \times 199 \times 153649 \times 599479 \times 33057806959$ \\
100 & $1.26765 \mathrm{E}+30$ & $\mathrm{~N}$ & 14 & $3 \times 5 \times 5 \times 5 \times 11 \times 31 \times 41 \times 101 \times 251 \times 601 \times 1801 \times 4051 \times 8101 \times 268501$ \\
& & & & $\rightarrow 3.5 \wedge 3.11 .31 .41 .101 .251 .601 .1801 .4051 .8101 .268501$ \\
\hline & & & & \\
\hline
\end{tabular}

Table 5. The Values of NPP ( $n=1$ to 50$)$ [Exponents are represented with ' $\wedge$ ']

\begin{tabular}{llll}
\hline$n$ & \multicolumn{1}{c}{$N P=2^{n-1}$} & \multicolumn{1}{c}{$\varphi(m)$} & \multicolumn{1}{c}{$N P P$} \\
\hline 1 & 1 & 0 & 0 \\
2 & 2 & 2 & 1 \\
3 & 4 & 6 & 2 \\
4 & 8 & 8 & 2 \\
5 & 16 & 30 & 6 \\
6 & 32 & 24 & 4 \\
7 & 64 & 126 & 18 \\
8 & 128 & 128 & 16 \\
9 & 256 & 432 & 48 \\
10 & 512 & 600 & 60 \\
11 & 1024 & 1936 & 176 \\
12 & 2048 & 1152 & 96 \\
13 & 4096 & 8190 & 630 \\
14 & 8192 & 10584 & 756 \\
15 & 16384 & 27000 & 1800 \\
16 & 32768 & 32768 & 2048 \\
17 & 65536 & 131070 & 7710 \\
18 & 131072 & 62208 & 3456 \\
19 & 262144 & 524286 & 27594 \\
20 & 524288 & 384000 & 19200 \\
& & &
\end{tabular}


Table 5. (Continued)

\begin{tabular}{llll}
21 & 1048576 & 1524096 & 72576 \\
22 & 2097152 & 2640704 & 120032 \\
23 & 4194304 & 8210080 & 356960 \\
24 & 8388608 & 4423680 & 184320 \\
25 & 16777216 & 32400000 & 1296000 \\
26 & 33554432 & 44717400 & 1719900 \\
27 & 67108864 & $1.13 \mathrm{E}+08$ & 4202496 \\
28 & 134217728 & 88510464 & 3161088 \\
29 & 268435456 & $5.34 \mathrm{E}+08$ & 18407808 \\
30 & 536870912 & $3.56 \mathrm{E}+08$ & 11880000 \\
31 & 1073741824 & $2.15 \mathrm{E}+09$ & 69273666 \\
32 & 2147483648 & $2.15 \mathrm{E}+09$ & 67108864 \\
33 & 4294967296 & $6.96 \mathrm{E}+09$ & $2.11 \mathrm{E}+08$ \\
34 & 8589934592 & $1.15 \mathrm{E}+10$ & $3.37 \mathrm{E}+08$ \\
35 & 17179869184 & $3.25 \mathrm{E}+10$ & $9.29 \mathrm{E}+08$ \\
36 & 34359738368 & $1.16 \mathrm{E}+10$ & $3.22 \mathrm{E}+08$ \\
37 & 68719476736 & $1.37 \mathrm{E}+11$ & $3.7 \mathrm{E}+09$ \\
38 & $1.37439 \mathrm{E}+11$ & $1.83 \mathrm{E}+11$ & $4.82 \mathrm{E}+09$ \\
39 & $2.74878 \mathrm{E}+11$ & $4.65 \mathrm{E}+11$ & $1.19 \mathrm{E}+10$ \\
40 & $5.49756 \mathrm{E}+11$ & $3.79 \mathrm{E}+11$ & $9.47 \mathrm{E}+09$ \\
41 & $1.09951 \mathrm{E}+12$ & $2.2 \mathrm{E}+12$ & $5.36 \mathrm{E}+10$ \\
42 & $2.19902 \mathrm{E}+12$ & $1.39 \mathrm{E}+12$ & $3.3 \mathrm{E}+10$ \\
43 & $4.39805 \mathrm{E}+12$ & $8.77 \mathrm{E}+12$ & $2.04 \mathrm{E}+11$ \\
44 & $8.79609 \mathrm{E}+12$ & $5.52 \mathrm{E}+12$ & $1.25 \mathrm{E}+11$ \\
45 & $1.75922 \mathrm{E}+13$ & $2.85 \mathrm{E}+13$ & $6.34 \mathrm{E}+11$ \\
46 & $3.51844 \mathrm{E}+13$ & $4.59 \mathrm{E}+13$ & $9.98 \mathrm{E}+11$ \\
47 & $7.03687 \mathrm{E}+13$ & $1.41 \mathrm{E}+14$ & $2.99 \mathrm{E}+12$ \\
48 & $1.40737 \mathrm{E}+14$ & $7.31 \mathrm{E}+13$ & $1.52 \mathrm{E}+12$ \\
49 & $2.81475 \mathrm{E}+14$ & $5.59 \mathrm{E}+14$ & $1.14 \mathrm{E}+13$ \\
50 & $5.6295 \mathrm{E}+14$ & $6.56 \mathrm{E}+14$ & $1.31 \mathrm{E}+13$ \\
\hline & & & \\
\hline 3
\end{tabular}

Table 6. The Values of NPP ( $n=51$ to 100) [Exponents are represented with ' $\wedge$ ']

\begin{tabular}{llll}
\hline$n$ & $N P-2^{n-1}$ & $\varphi(m)$ & $N P P$ \\
\hline 51 & $1.1259 \mathrm{E}+15$ & $1.91 \mathrm{E}+15$ & $3.75 \mathrm{E}+13$ \\
52 & $2.2518 \mathrm{E}+15$ & $2.34 \mathrm{E}+15$ & $4.5 \mathrm{E}+13$ \\
53 & $4.5036 \mathrm{E}+15$ & $9.01 \mathrm{E}+15$ & $1.7 \mathrm{E}+14$ \\
54 & $9.0072 \mathrm{E}+15$ & $2.85 \mathrm{E}+15$ & $5.28 \mathrm{E}+13$ \\
55 & $1.80144 \mathrm{E}+16$ & $3.29 \mathrm{E}+16$ & $5.99 \mathrm{E}+14$ \\
56 & $3.60288 \mathrm{E}+16$ & $3.35 \mathrm{E}+16$ & $5.99 \mathrm{E}+14$ \\
57 & $7.20576 \mathrm{E}+16$ & $1.24 \mathrm{E}+17$ & $2.17 \mathrm{E}+15$ \\
58 & $1.44115 \mathrm{E}+17$ & $1.88 \mathrm{E}+17$ & $3.24 \mathrm{E}+15$
\end{tabular}


Table 6. (Continued)

\begin{tabular}{|c|c|c|c|}
\hline & 17 & 17 & \\
\hline & $5.76461 \mathrm{E}+17$ & $2.17 \mathrm{E}+17$ & $3.61 \mathrm{E}+15$ \\
\hline & 8 & +18 & \\
\hline & 8 & 18 & 6 \\
\hline & $4.61169 \mathrm{E}+18$ & $.61 \mathrm{E}+18$ & +17 \\
\hline & +18 & $21 \mathrm{E}+18$ & $\mathrm{~F}+17$ \\
\hline & $1.84467 \mathrm{E}+19$ & 19 & \\
\hline & $5 \mathrm{E}+19$ & $61 \mathrm{E}+19$ & $06 F_{+}$ \\
\hline & 7.3 & 20 & \\
\hline & +20 & & \\
\hline & +20 & $95 \mathrm{E}+20$ & 710 \\
\hline & 5.9 & 20 & \\
\hline & +21 & & \\
\hline & $236118 \mathrm{~F}$ & 7465,20 & 104 \\
\hline & $4.72237 \mathrm{E}+21$ & $.42 \mathrm{E}+21$ & $1.29 \mathrm{E}-$ \\
\hline & $9.44473 \mathrm{E}+21$ & $1.25 \mathrm{E}+22$ & 1.6 \\
\hline & 1.8889 & $3.11 \mathrm{~L}+22$ & $4.14 \mathrm{E}+2 \mathrm{U}$ \\
\hline & 3.777 & Lis & 5 \\
\hline & $7.55579 \mathrm{E}+22$ & $1.42 \mathrm{E}+23$ & 1.84 \\
\hline & 1.51 & .14 & 1.4 \\
\hline & $302231 \mathrm{~F}+23$ & $6.04 \mathrm{E}+23$ & $7.65 \mathrm{E}+21$ \\
\hline & 6.04 & $4.15 \mathrm{E}+23$ & $E+21$ \\
\hline & .2 & 4 & $2.52 \mathrm{E}+22$ \\
\hline & 24178 & 4 & $\mathrm{OF}$ \\
\hline & 4.835 & +24 & +23 \\
\hline & 9.6714 & $.31 E+24$ & 5. \\
\hline & 10240 & $374 \mathrm{~F}+25$ & ( \\
\hline & $3.86856 \mathrm{E}+25$ & $5.15 \mathrm{E}+25$ & $5.98 \mathrm{E}+23$ \\
\hline & $7.73713 \mathrm{H}$ & $1.32 \mathrm{E}+20$ & 1.52 \\
\hline &  & $1.46 \mathrm{E}+26$ & 24 \\
\hline & $309485 \mathrm{~F}+26$ & $6.19 \mathrm{E}+26$ & $6.95 \mathrm{E}+24$ \\
\hline & $6.1897 \mathrm{E}+26$ & $2.56 \mathrm{E}+26$ & $2.84 \mathrm{E}+24$ \\
\hline & $1.23794 \mathrm{E}$ & $2.45 \mathrm{E}+21$ & $2.7 \mathrm{~L}+2 \mathrm{~J}$ \\
\hline & $2.47588 \mathrm{E}+27$ & $2.57 \mathrm{E}+27$ & $2.79 \mathrm{E}+25$ \\
\hline & $4.95176 \mathrm{E}+27$ & $8.49 \mathrm{E}+27$ & $9.13 \mathrm{E}+25$ \\
\hline & .90 & 1 & 1.4 \\
\hline & 1.9807 & $3.81 \mathrm{E}$ & 4.0 \\
\hline & $3.96141 \mathrm{E}+28$ & $2.05 \mathrm{E}+28$ & $2.13 \mathrm{E}+26$ \\
\hline & 7.92 & 9 & 1. \\
\hline & 1.58 & $2.05 \mathrm{E}+29$ & $2.09 \mathrm{E}+27$ \\
\hline & $3.16913 \mathrm{E}+29$ & $5.04 \mathrm{E}+29$ & $5.09 \mathrm{E}+27$ \\
\hline & $6.33825 E+29$ & $3.65 E+29$ & $3.65 \mathrm{E}+27$ \\
\hline
\end{tabular}


the generators need to be used or not. Hence these tables are of great help for engineers, scientists and researchers practicing / working their skills in the fields of DSSS, BIST and data security.

\section{Acknowledgement}

The acknowledgements are due to authorities of Sultan Qaboos University (Sultanate of Oman) for providing generous research support grants and environments for carrying out the research works.

\section{References}

1. Ahmad A (2012). Better PN generators for CDMA application - a Verilog-HDL implementation approach, International Journal of Information Engineering (IJIE), vol 2(1), 6-11.

2. Ahmad A, Al-Abri D et al. (2012). Adding pseudo-random test sequence generator in the test simulator for DFT approach, Journal of Computer Technology and Applications (JCTA), vol 3(7), 463-470.

3. Ahmad A, and Hayat L (2011). Selection of polynomials for cyclic redundancy check for the use of high speed embedded - an algorithmic procedure, Transactions on Computers (WSEAS), vol 10(1), 16-20.

4. Ahmad A (2010). A simulation experiment on a built-in self test equipped with pseudorandom test pattern generator and Multi-Input Shift Register (MISR), International Journal of VLSI Design \& Communication Systems, vol 1, No. 4, 1-12.

5. Ahmad A, and Al-Balushi J (2009). How to design an effective Serial Input Shift Register (SISR) for data compression process of built-in self-test methodology, Proceedings 4th International Design and Test Workshop (IDT'09) held at King Abdul Aziz City for Science and Technology (KAASAT), Riyadh, Saudi Arabia. 372-379.

6. Al-Naamany A M, and Ahmad A (2003). Development of a strong stream ciphering technique using non-linear fuzzy logic selector, Mobile and Wireless Communications, IFIP The International Federation for Information Processing, vol 106, 199-206.

7. Ahmad A, Al-Musharafi M J et al. (2002). Design and study of a strong stream crypto-system model for e-commerce, Proceeding ICCC ' 02 Proceedings of the 15th International Conference on Computer Communication, vol 1, 619-630.

8. Jamil T, and Ahmad A (2002). An investigation in to the application of linear feedback shift registers for steganography, Proceedings IEEE SoutheastCon2002, Columbia, SC, USA, 239-244.
9. Ahmad A, Al-Musharafi M J et al. (2001). An NLFSR based sequence generator for stream ciphers, Proceedings (SETA'01) - An International Conference on Sequences \& Their Applications, Norway (Bergen), 11-13.

10. Golic J D (2000). Cryptanalysis of three mutually clockcontrolled stop/go shift registers, IEEE Transactions on Information Technology, vol 46, No. 3, 1081-1090.

11. Ahmad A (1997). Achievement of higher testability goals through the modification of shift register in LFSR based testing, International Journal of Electronics (UK), vol 82, No. 3, 249-260.

12. Chen H W, Aine C J E et al. (1996). Nonlinear analysis of biological systems using short $\mathrm{m}$-sequences and sparse-simulation techniques, Annals of Biomedical Engineering, vol 24, 513-536.

13. Golomb S W (1982). Shift Register Sequences, Aegean Park Press, Revised Edition.

14. Ahmad A, Nanda N K et al. (1990). Are primitive polynomials always best in signature analysis?, IEEE Design \& Test of Computers (USA), vol 7, No. 4, 36-38.

15. Blum L, Blum M et al. (1986). A simple unpredictable pseudo-random number generator, SIAM Journal of Computing, vol 15, No. 2, 364-383.

16. Ahmad A, Nanda N K et al. (1988). A critical role of primitive polynomials in an LFSR based testing technique, IEE Electronics Letters (UK), vol 24(15), 953-955.

17. Ahmad A, Al-Busaidi S S et al. (2013). Study on cyclic crosscorrelation behavior of maximal length pseudo-random binary sequences, Indian Journal of Industrial and Applied Mathematics (Taylor \& Francis), vol 4(1), 33-43.

18. Ahmad A, and Al-Abri D (2012). Design of a pseudo-random binary code generator via a developed simulation model, International Journal on Information Technology (ACEEE Journal), vol 2(1), 33-36.

19. Ahmad A (2011). Investigation of some quite interesting divisibility situations in a signature analyzer implementation, Transactions on Circuits and Systems (WSEAS), vol 10 (9), 299-308.

20. Ahmad A (1998). An algorithmic generation of sparse primitive polynomials of order $n$, International Wireless and Telecommunication Symposium (IWATS'98), Shah Alam (Malaysia).

21. Ahmad A, and Elabdalla A M (1997). An efficient method to determine linear feedback connections in shift registers that generate maximal length pseudo-random up and down binary sequences, Computer \& Electrical Engineering (Elsevier), vol 23(1), 33-39.

22. Ahmad A, and Al-Maashri A (2008). Investigating some special sequence length generated through an external exclusive-NOR type LFSRs, International Journal Electrical and Computer Engineering, vol 34(1), 270-280. 
23. Ahmad A (2003). Realization of a stream cipher with better security and higher reliability goals, 2nd International Conference on Quality, Reliability and Information Technology - Trends and Future Directions, 18-21, 77-78.

24. Ahmad A, Al-Busaidi S et al. (2003). Measurement techniques of LFSR sequences, Proceedings International Symposium on Wireless Systems and Networks (ISWSN'03), King Fahad University of Petroleum - Dahran, Kingdom of Saudi Arabia, 1-5.

25. Ahmad A, Nanda N K et al. (1989). The use of irreducible characteristic polynomials in an LFSR based testing of digital circuits, Proceedings of 4th IEEE International Conference of Region 10 (TENCON-89), 494-496.

26. Ahmad A, Ahmed Al-Mashari et al. (2009). On locking conditions in $\mathrm{M}$-sequence generators for the use in digital watermarking, Proceedings International Conference on Methods and Models in Computer Science (ICM2CS09) held at School of Computer \& Systems Sciences, Jawaharlal Nehru University, New Delhi, India, 111-115.

27. Ahmad A, Al-Musharafi M J et al. (2001). Study and implementation of properties of $\mathrm{m}$-sequences in MATLABSIMULINK - A pass / fail test tool for designs of random generators, Proceedings IEEE / IEE International Conference on Communication, Computer and Power (ICCCP'01), Oman, 191-196.

28. Ahmad A, Al-Musharafi M J et al. (2002). Study and implementationofpropertiesofm-sequencesinMATLAB-SIMULINK - A pass / fail test tool for designs of random generators, Journal of Scientific Research - Science and Technology, vol 7 (part 1), $147-156$

29. Al-Lawati A, and Ahmad A (2004). Realization of a simplified controllability computation procedure - a MATLAB-SIMULINK based tool, Sultan Qaboos University Journal for Scientific Research - Science and Technology, Oman, vol 8, 131-143.

30. Ahmad A, and Ruelens D (2013). Development of digital logic design teaching tool using MATLAB \& SIMULINK, IEEE Technology and Engineering Education (ITEE), vol 8, No. 1, 7-12.

31. Ahmad A, Ruelens D et al. (2013). Development of verification tool for minimal Boolean equation, IEEE Technology and Engineering Education (ITEE), vol 8, No. 3.

32. MATLAB: Available from: http://www.mathworks.com/ 